\title{
Implementation of Real Time Remote Water Quality Monitoring System Using Wireless Sensors and Arduino Based Data Logger
}

\author{
Goib Wiranto ${ }^{+}$, Yudi Y. Maulana, Iqbal Syamsu and Dayat Kurniawan \\ Research Center for Electronics and Telecommunication, Indonesian Institute of Sciences (LIPI), Jl. \\ Sangkuriang, Bandung 40135, Indonesia
}

\begin{abstract}
The design and implementation of a Wireless Sensor Network (WSN) for real time remote water quality monitoring system will be described in this paper. The main system consists of a wireless sensing node, an arduino based data logger with onsite display, and a web based data server. The wireless sensing node was used to collect information on the water quality parameters, that is $\mathrm{DO}, \mathrm{pH}$, and temperature, and send the measured parameter values to the data logger using Xbee wireless transmitter. Central to the system is the data logger, where data will be displayed and transmitted to the web based server using GPRS protocol. The system has been implemented to monitor the water quality parameters at a shrimp aquaculture in Bangka island, and data have been continuously collected by web server in Bandung, West Java for several months.
\end{abstract}

Keywords: water quality; online monitoring; sensors; database server; data logger

\section{Introduction}

In developing countries such as Indonesia, managing water resources has become a fundamental issue that has been discussed seriously by the government and public organisations over the past decade. The aim was to ensure sustainability of water resources for future generation. Water resource management not only concerns about water conservation, but also includes providing information about water quality, that should be accessible by the public so that early preparation can be taken by those affected by degradation in water condition [1]. Such information can be provided only by a well planned water quality measuring network applied to specific uses such as drinking [2], industrial [3], agriculture [4], aquaculture [5], etc. The main problem in realising a network of water quality monitoring system in island countries like Indonesia is related to geographical location separating the water resources and the management. In the past, geographical problems were solved by manual data collection relying on human ability to collect samples and subsequently analyse them in the laboratory. This process was not only time consuming, but also expensive and low in time resolution.

But with the advance in Information Technology, now data can be collected onsite and transmitted across wide areas using network of wireless sensors. It is not surprising that over the past few years, Wireless Sensor Network (WSN) has received considerable attention from academia as well as industries because of its widespread applications [6]. The main advantage of WSN is its low implementation and maintainance cost, because the setup does not require fix infrastructures. In addition, WSN requires low power [7], and can be installed in any places which are difficult to reach and remotely isolated, covering a wide area of different types of water resources [8]. These advantages have attracted environmental researchers in applying WSN to monitor environmental condition including water quality parameters [9].

Previous works demonstrating the use of WSN for water quality monitoring have been shown by Faustine et al. [10] to monitor Lake Victoria Basin. The system, consisting of a sensor node, a gateway node,

${ }^{+}$Corresponding author. Tel.: +62 8170217746 .

E-mail address:gwiranto@gmail.com. 
and software application, was capable of delivering four water quality parameters (temperature, $\mathrm{DO}, \mathrm{pH}$, and conductivity) to relevant stakeholders using web based and mobile phone platforms. Similarly, Jiang et al. [11] developed a WSN suitable for complex and large scale water resource monitoring, whereas Yang et al. [12] showed the WSN design suitable for a long term application in aqueous environment. In most recent WSN architectures, the trend has been using a custom data logging system to acquire environmental quality data and transmitting it to a remote data server, by which then the data will be distributed to a website or mobile phone devices [13,14].

In this paper, the design and implementation of a WSN to monitor water quality parameters will be described. The system consists of sensors to monitor water quality parameters ( $\mathrm{DO}, \mathrm{pH}$, and temperature), an Arduino based data logger, and a database server with software application. The system has been tested in the field to monitor the water quality of a shrimp aquaculture and capable of delivering real time data for long term usage.

\section{System Design and Structure}

The system has been designed on a modular basis to allow for easy maintainance and flexibility for further development or modification. As can be seen in Figure 1, the main structure consisted of a sensing node, a data logger, and a web based data server. The sensing node has the function of performing initial data collection about the water quality parameters from the location of installation. In this case, three water quality parameters of interest are $\mathrm{DO}, \mathrm{pH}$, and temperature. Data from the sensing node are transmitted to the data logger using Xbee wireless transmitter. The data logger then processed, displayed, and transmitted the data to the data server using GPRS transmission. At the server, the data will be uploaded to a website and sent to some designated mobile phones with Android applications.

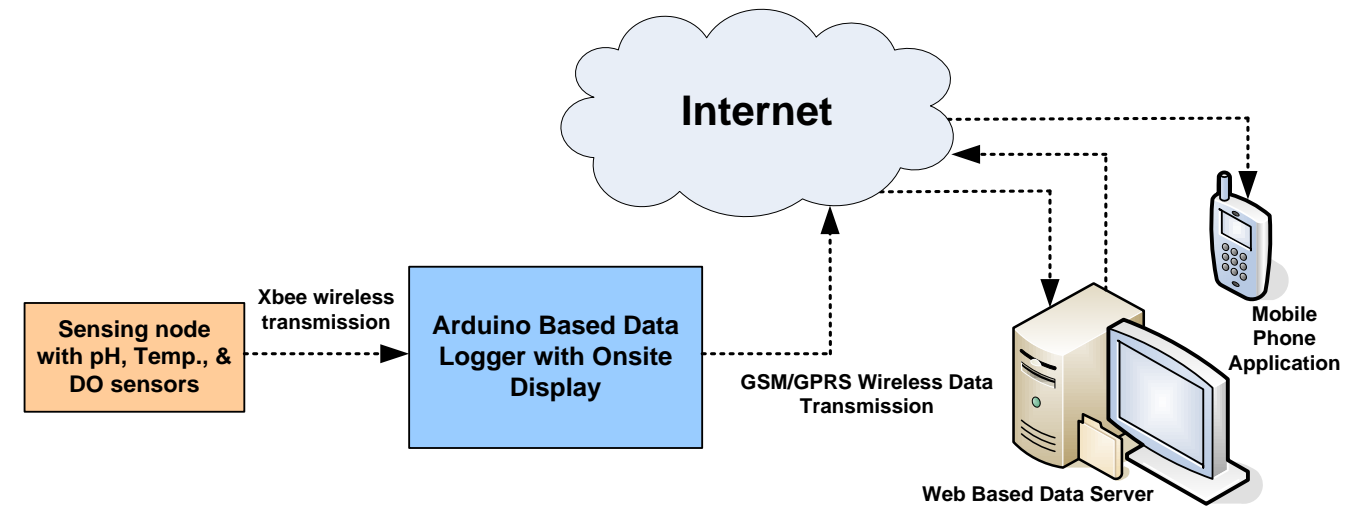

Fig. 1: The structure of wireless sensor network system developed using sensing node, Arduino based data logger, and web based data server.

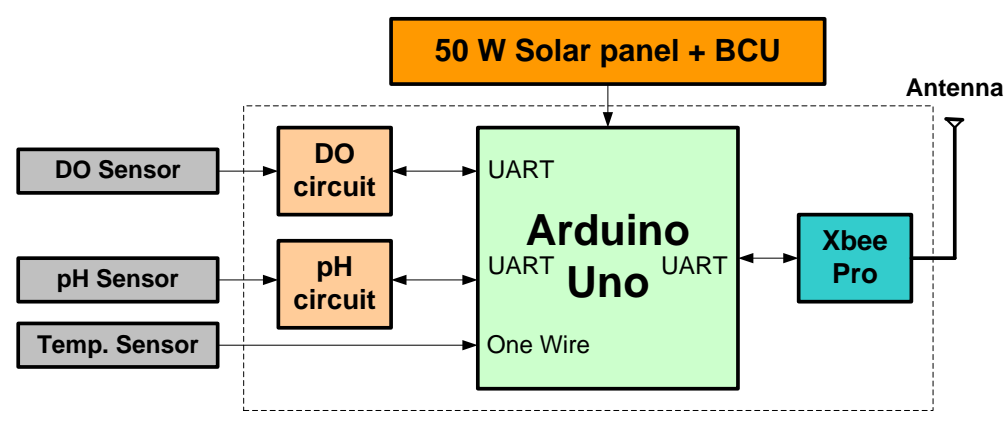

(a)

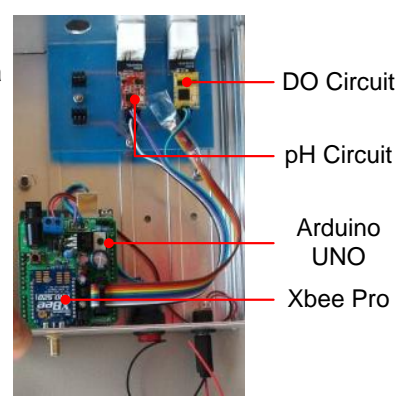

(b)

Fig. 2: (a). Block diagram of the sensing node, (b). Component layout inside an aluminium case

\subsection{The sensing node}

The sensing node is the frontline of the entire WSN system. It consisted of three water quality sensors, interface ciruits, an Arduino Uno microcontroller, and an Xbee Pro wireless transmitter. The sensing node 
module has been powered by a $50 \mathrm{~W}$ solar panel, and thus it can be installed in almost any location.The $\mathrm{pH}$ sensor used was from Atlas Scientific with the probe sensitivity range from 0 - 14. The DO sensor was also from Atlas Scientific with probe sensitivity range of $0-20 \mathrm{mg} / \mathrm{l}$. The temperature sensor was from Maxim Integrated type DS18B20 with a measurement range of -55 to $125^{\circ} \mathrm{C}$. The block diagram of the entire sensing node can be seen in Figure 2. The pH and DO circuits were required to convert the outputs of both sensors into digital format to be readable by the Arduino microcontroller. Operation of the sensing node was performed by the Arduino microcontroller using a set of instructions from initiating the hardware and software connection to collecting and transmitting the sensor data using Xbee transmitter.

\subsection{The arduino based data logger}

The main unit of the data logger is an Arduino Mega, which is used to control communication from and to the sensing node using an Xbee Pro, data transmission to the server using GSM/GPRS module (SIM908), and data display using LCD 16X2. The Xbee Pro module is used to receive the data transmitted from the sensing node. Once received, the data will be immediately displayed on the LCD along with the date and time data from the RTC DS1307 (Real Time Clock). The RTC module was accessed via I2C protocol of the Arduino Mega. All data (sensor and time data) are then transmitted to the data server using SIM908 module at 10 min interval. Figure 3 shows the block diagram and realisation of the data logger components.

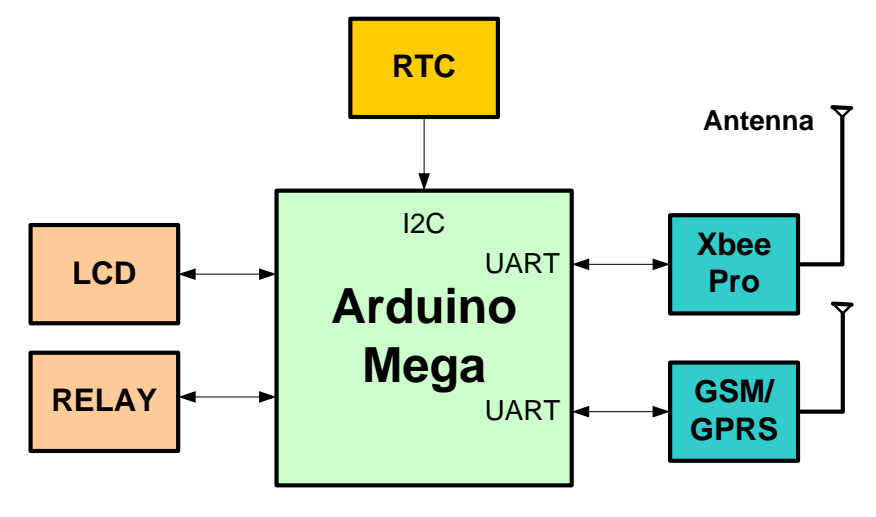

(a)

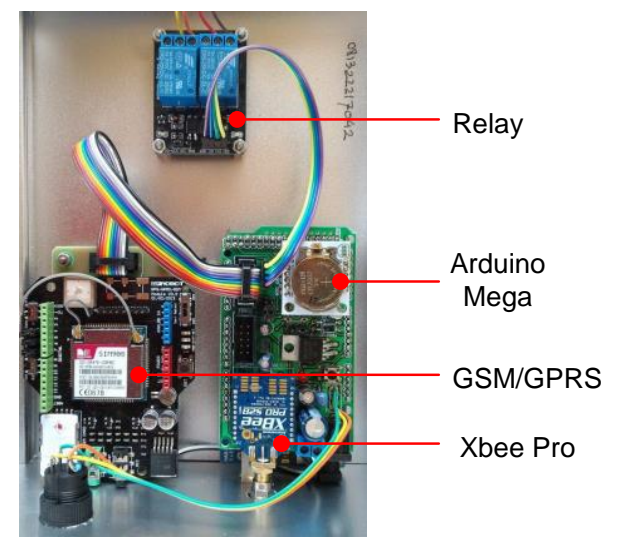

(b)

Fig. 3: (a). Block diagram of the Arduino based data logger, (b). Realisation of the data logger components

\subsection{The database server node}

At the database server, a software application has been developed to store the water quality data and visually display the data in tabular or graphical format.The database was developed using mySQL, and the structure was designed to allow data from multiple sensing nodes to be collected, anticipating for future system development. Data quarying from the database was performed using PHP based web programming, by which data can be sent to several designated mobile phones with WhatsApps and Twitter applications.

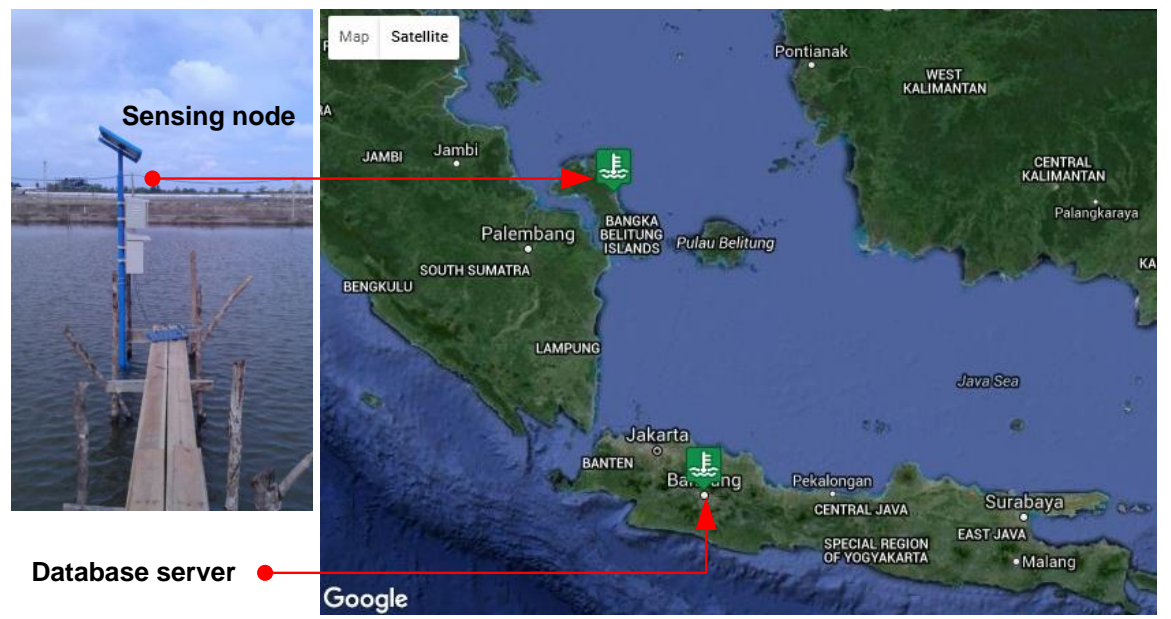

Fig. 4: Location of the sensing node in Bangkaisland and the database server in Bandung, West Java. 


\section{Implementation, Results, and Discussion}

The system has been implemented to measure the water quality parameters of a shrimp aquaculture located in Bangka island, and the database server is located in Bandung, West Java. As can be seen in Figure 4 , the sensing node was installed in the middle of a shrimp pond, using a $50 \mathrm{~W}$ solar panel as the power source.The data logger was placed on the side of the pond within $30 \mathrm{~m}$ distance from the sensing node. Since its first installation in November 2015, the system has been continuously sending data to the database server without any significant interuption, except during maintenance period. This means that the system has proved itself suitable for a long term, outdoor monitoring application.

In Figure 5 (left), it can be seen the website view of the water quality data which is updated every 10 minute interval. This information is accessible by the public from http://www.ppet.lipi.go.id/water/home.php, however, for the historical and graphical display of the data, it requires a login access into the system. In Figure 5 (right), the screen shot view of the data as appeared on a mobile phone's Whatapps application is shown. The data on the mobile phone application was also updated every 10 minutes. Several mobile phone numbers have been selected belonging to the management of the aquaculture bussiness to receive information of the water quality parameters.

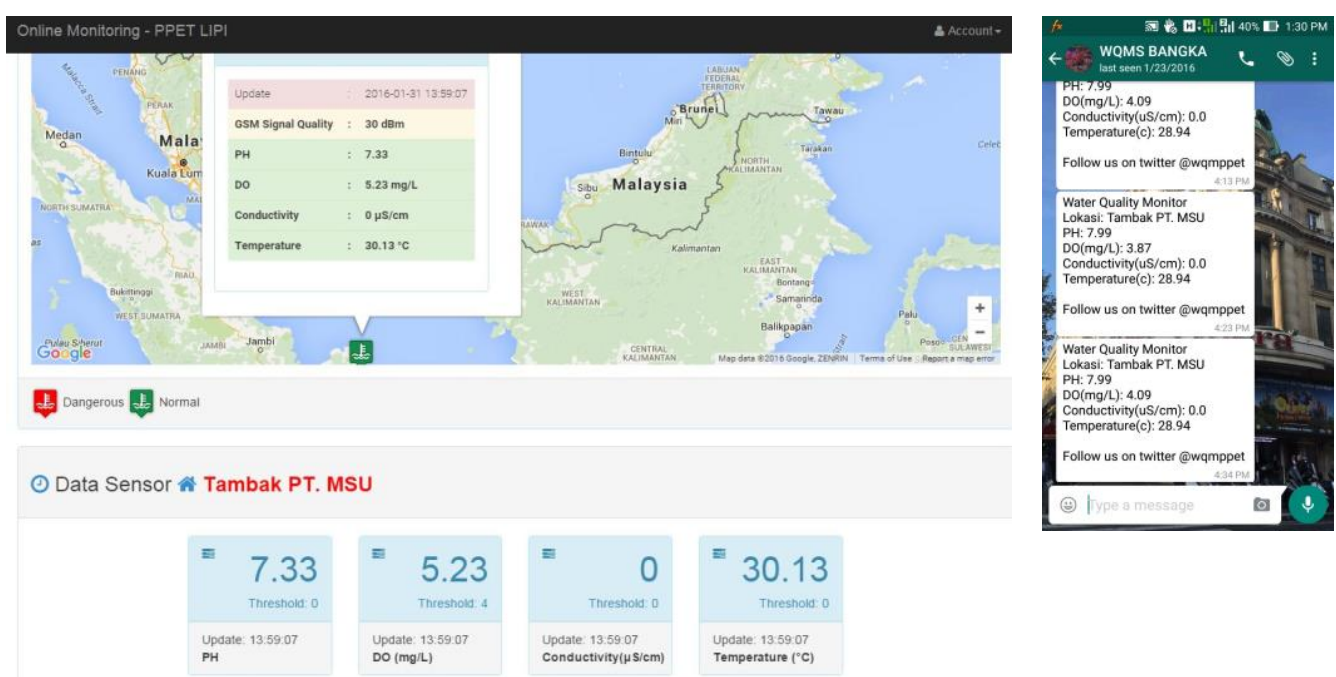

Fig. 5: Screenshot of website view (left) and mobile phone Whatapps application (right)

In Figure 6, a typical graphical view of the water quality parameter data is shown, with the period of 24 hours for parameter DO and pH.The data was taken on December 30, 2015. It can be seen that the DO values were high during the afternoon and low in the morning and at night time, which is normal due to the increase in photosynthesis activitiy of the algae in the ponds. When the DO value was low, usually an aeration system was turned on to increase the DO value.

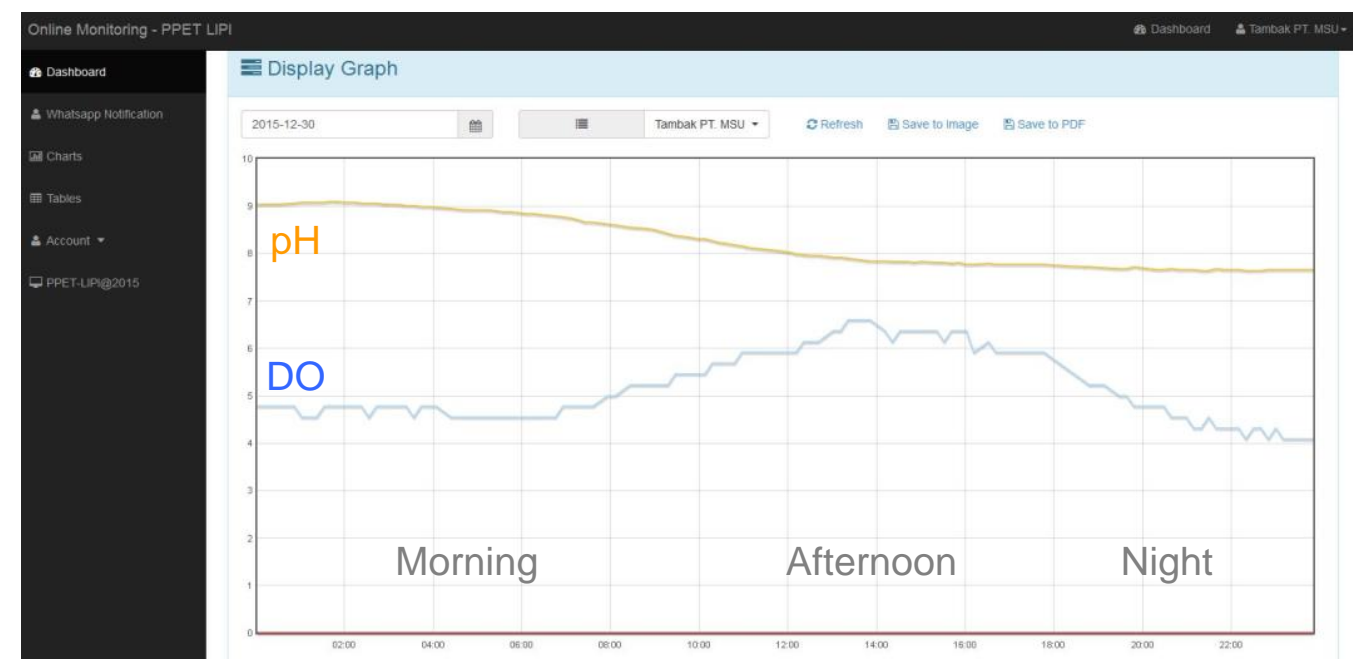

Fig. 6: Graphical display of DO and pH parameters taken from the web based data server 


\section{Conclusion}

This paper has described the design and implementation of a WSN for real time remote water quality monitoring system consisting of a wireless sensing node, an arduino based data logger with onsite display, and a web based data server. The sistem has been implemented to monitor the water quality parameters, DO, $\mathrm{pH}$, and temperature, at a shrimp aquaculture in Bangka island, and data have been continuously collected by web server in Bandung for several months. By accessing the website at http://www.ppet.lipi.go.id/water/home.php, the public can get live information on the water quality parameters. The developed system has proved itself suitable for a long term, outdoor water quality monitoring application. In the future, several sensing nodes will be developed and installed in different locations and integrated with the current system to form a wide area WSN.

\section{Acknowledgements}

This work was financially supported by Indonesian Institute of Sciences (LIPI) under the scheme of the Implementation of Science and Technology to Society (Iptekda).

\section{References}

[1] D. Hou, X. Song, G. Zhang, H. Zhang, and H. Loaiciga. An early warning and control system for urban, drinkingwater quality protection: China's experience. Environ. Sci. Pollut. Res. 2013,20:4496-4508.

[2] D. D. Ediriweera and I.W. Marshall. Monitoring water distribution systems: Understanding and managing sensor networks. Drink. Water Eng. Sci. 2010,3: 107-113.

[3] Y. Zhang, W. Yang, D. Han, and Y. -I. Kim. An integrated environment monitoring system for underground coal mines—wireless sensor networksubsystem with multi-parameter monitoring. Sens. 2014, 14: 13149-13170.

[4] A.Gaddam, M. Al-Hrooby, and W. F.Esmael. Designing a wireless sensors network formonitoring and predicting droughts. Proc. 8th. Int. Conf. Sens. Tech. Liverpool: 2014, pp. $210-215$.

[5] K.G.Sutar and R.T.Patil. Wireless sensor network system to monitor the fish farm. Int. J. of Eng. Res. and App. 2013, 3 (5): $194-197$.

[6] C.Albaladejo, P. Sánchez, A.Iborra, F. Soto, J. A. López,andR. Torres. Wireless sensor networks for oceanographic monitoring:a systematic review. Sens. 2010, 10: 6948-6968.

[7] R.Yue and T. Ying. A novel water quality monitoring system based on solarpower supply \& wireless sensor network. Proce. Env. Sci. 2012,12: 265 - 272.

[8] W. -Y. Chung and J. -H.Yoo. Remote water quality monitoring in wide area. Sens. Act. B. 2015, 217: 51-57.

[9] G.Xu, W.Shen, and X. Wang. Applications of wireless sensor networks in marineenvironment monitoring: a survey. Sens. 2014, 14, 16932-16954.

[10] A.Faustine, A. N. Mvuma, H. J. Mongi, M. C. Gabriel, A. J. Tenge,and S. B. Kucel. Wireless sensor networks for water quality monitoring and control within lake Victoriabasin: prototype development. Wireless Sens. Net., 2014, 6: $281-290$.

[11] P. Jiang, H. Xia, Z. He, and Z. Wang. Design of a water environment monitoring system based onwireless sensor networks. Sens., 2009, 9: 6411-6434.

[12] X. Yang, K. G. Ong, W. R. Dreschel, K.Zeng, C. S. Mungle, and C. A. Grimes. Design of a wireless sensor network for long-term, in-situmonitoring of an aqueous environment. Sens. 2002, 2: 455-472.

[13] D. Sirisha, B. Venkateswaramma, M. Srikanth, and A. A. Babu. Wireless sensor based remote controlled agriculture monitoring system using zigbee. SSRG Int. J. Elec. Com. Eng. 2015, 2 (4): 32 - 36.

[14] N. Gahlot, V. Gundkal, S. Kothimbire, and A. Thite. Zigbee based weather monitoring system. The Int. J. Eng. Sci. 2015, 4 (4): $61-66$. 